\title{
The TanDEM-X Mission Operations Segment: Close formation flight: Preparation and First Experiences
}

\author{
H. Hofmann ${ }^{1}$ \\ DLR, 82234 Wessling, Germany \\ and \\ R. Kahle ${ }^{2}$ \\ DLR, 82234 Wessling, Germany
}

\begin{abstract}
In 2007 the TerraSAR-X satellite was successfully launched. Its payload consists of an earth observing Synthetic Aperture Radar, which supplies high resolution radar images. The yet to be launched TanDEM-X mission extends the TerraSAR-X mission with a second satellite of very similar capabilities. The two satellites will be flying in close formation $(250 \mathrm{~m}-500 \mathrm{~m})$, such that the radar instruments can be synchronized (bistatic mode). This bistatic mode allows the generation of three dimensional pictures and thus a high resolution elevation model of the observed area (according to HRTI-3 specifications), which in the end should cover the whole earth.

TanDEM-X is the first project worldwide to fly a close formation with a minimum distance of only a few hundred meters. During the development and integration of the ground segment, it became obvious that the amount of security measures have to be significantly increased to ensure safe operations. One of the main influences is the satellite orbit and attitude control system that is based on active control via hydrazine thrusters. The activation of the thrusters for attitude control possibly also changes the orbit, which leads to a change of the relative positions. Consequently two problems can appear, either mutual radar illumination which might damage electronic equipment or if the impulse is large enough, a collision.

The paper gives an overview of how the various elements of the TanDEM-X ground segment, with main emphasis on the mission operations segment, had to be designed to provide a maximum degree of safety. This includes all aspects of operations, from ground station network and flight procedures to extended on-call services and specifically developed tools. It is shown how the operational design is influenced by the satellite design and how a different satellite design would have reduced the operational effort.

It was originally planned to present the first real experiences with both satellite flying in close formation, but due to a launch delay to end of May 2010 this is unfortunately not possible.
\end{abstract}

\section{Nomenclature}

$\Delta v \quad=$ Velocity increment due to thruster activity

$e \quad=$ Eccentricity of the satellite's orbit

$i=$ Inclination of the satellite's orbit; both $e$ and $i$ are treated as vectors by combining the numerical value with the argument of perigee and the right ascension of the ascending node

\footnotetext{
${ }^{1}$ TerraSAR-X/TanDEM-X Project Manager, German Space Operations Center, DLR Oberpfaffenhofen, 82230 Wessling, Germany/ Harald.Hofmann@dlr.de

${ }^{2}$ TerraSAR-X/TanDEM-X Flight Dynamics Manager, German Space Operations Center, DLR Oberpfaffenhofen, 82230 Wessling, Germany/ Ralph.Kahle@dlr.de
} 


\section{Introduction}

TanDEM-X stands for TerraSAR-X add-on for Digital Elevation Measurement and consists of a pair of nearly identical satellites with the primary mission goal to generate a highly precise digital elevation model (DEM) of the complete surface of the earth with an accuracy better than $10 \mathrm{~m}$. The main payload to perform this task is a synthetic aperture radar (SAR) on both satellites that allows high resolution images up to approx. $1 \mathrm{~m} * 1 \mathrm{~m}$ pixel size. By synchronizing both SAR antennas, it is possible to generate interferometric pictures that in turn allow the production of the digital elevation model. To achieve the required elevation accuracy and to be able to synchronize the SAR antennas, the relative distance between both satellites has to be very small, from approx. only $200 \mathrm{~m}$ up to $2000 \mathrm{~m}$.

The first satellite TerraSAR-X (TSX) was launched in June 2007, and the first SAR images were taken and processed after only 5 days in orbit. Since then, TSX operations have been running very smoothly with a constant high quality of data products.

The second satellite (TDX) of the TanDEM-X mission was planned for launch in October 2009. Unfortunately, the launch has been delayed until end of May 2010 due to unexpected problems with the radar hardware. Like TSX, TDX will be launched from Baikonur into a sun-synchronous dusk-dawn orbit with an altitude of about $515 \mathrm{~km}$. TDX will be controlled with respect to TSX in a so-called helix formation with one complete cycle during one orbit, whereas TSX is controlled in a $\pm 250 \mathrm{~m}$ tube around a prescribed earth-fixed reference orbit, leading to an eleven day repeat cycle. The helix formation allows the adjustment of different baselines (i.e. basically the relative distance between TSX and TDX) which are directly influencing the DEM accuracy. The stability of the formation is achieved by so-called e-,i-vector separation (relative eccentricity / relative inclination, whereby maximum $\Delta \mathrm{i}$ occurs at the equator and maximum $\Delta$ e near the poles; see $^{2}$ ). Figure 1 gives a graphical overview:
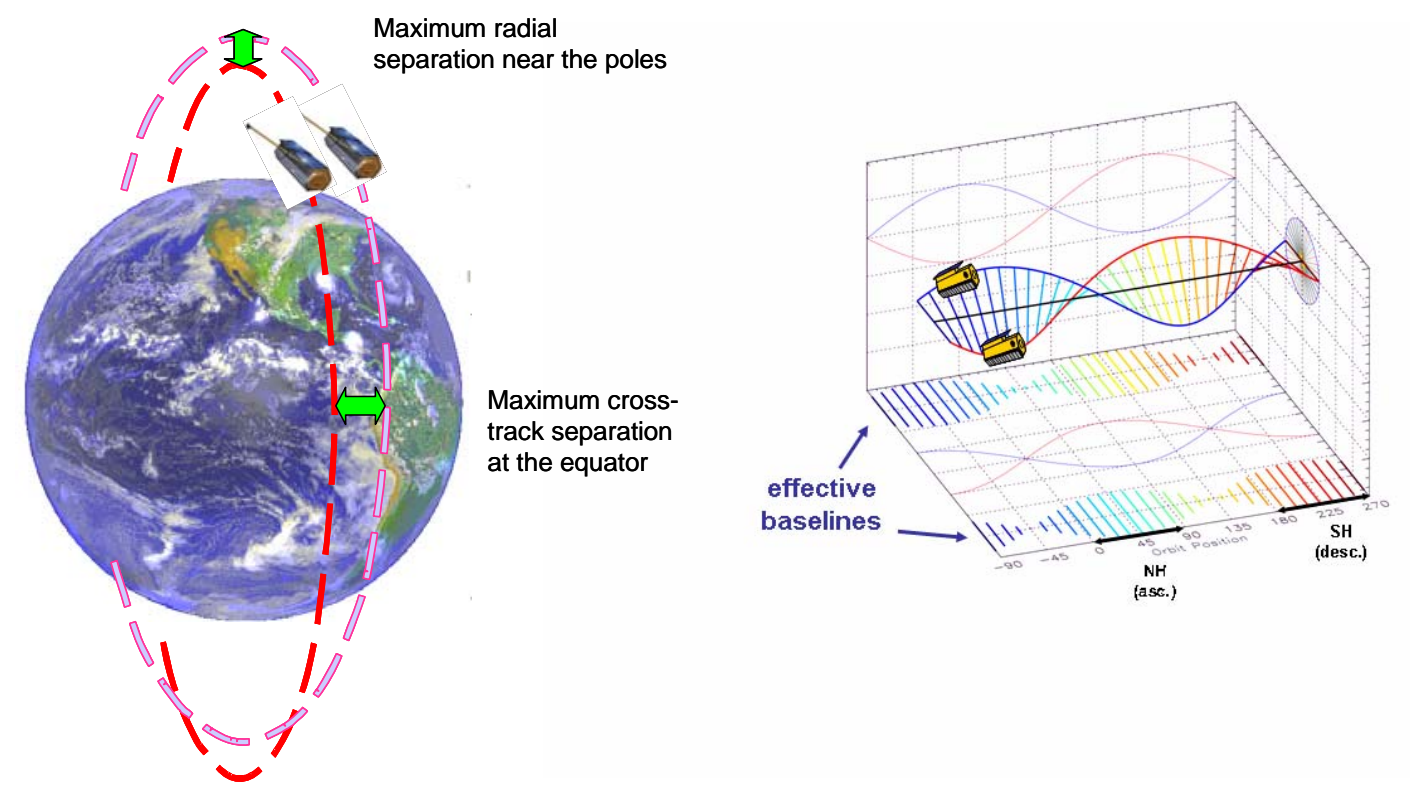

Figure 1. Graphical illustration of the helix formation. During one orbital revolution TDX circles TSX with maximum cross-track separation at the equator and maximum radial separation near the poles. The picture on the right hand side shows how the effective baselines change during one orbit $\left(\right.$ from $\left.{ }^{4}\right)$. 
As described above, the minimum distance will be only about $200 \mathrm{~m}$, which from the beginning of the project raised the question about how safe such a formation can be with respect to collision. As a consequence the e-i-vector separation concept was introduced providing passive formation stability for at least two weeks during nominal mission $\left(\mathrm{see}^{2}\right)$. However, the design of the thruster based satellite safe mode, which is triggered in case of major problems (sensor problems, large attitude deviations or similar problems), is such that significant perturbations of the orbit are accepted in favour of quick stabilisation. In-flight experience with TSX safe modes also showed that distances of several hundred meters were easily exceeded. As consequence, the thruster based safe mode would suspend the passive formation stability within only a few hours. A new concept had to be developed.

\section{Safe formation flight}

After evaluating the safe modes experienced on TSX, the general problem - particularly with respect to the TanDEM-X mission - was identified quickly. In principle, two problem cases can appear: either illumination by the active radar of the other satellite, possibly damaging the electronics, or as absolute worst case a collision. Still both problems can only appear if the orbit is disturbed. In other words, as long as there are no unexpected thruster activities (maneuvers have to be considered differently), and therefore no unexpected orbit change of either satellite, there is no collision danger. Unfortunately, the safe mode was precisely based on the thrusters, therefore in any severe attitude problem the satellites would just use them and change their orbit unpredictably. While this was fine for a single satellite it became a problem for the formation. The satellite safe mode, designed to bring the satellite back to a nominal attitude as quick as possible, had become a potentially un-safe mode.

Consequently, one of the first actions was to discuss with the satellite manufacturer, if a different safe mode without changing the orbit could be established. The solution was the introduction of an additional safe mode based upon the magnetic torque rods (ASM-MTQ) instead of the propulsion system (for details see ${ }^{1}$ ). Since the torque rods only influence the attitude, this concept allows the satellite to survive in safe mode until the ground has time to identify the problem and recover the satellite without having to fear a potential collision. Further discussions revealed additional elements that needed either to be changed or newly developed to complement the on-board measures to ensure formation safety and shorten reaction times. Figure 2 gives an overview of the overall concept which will be described in more detail in following sections, with emphasis on the ground aspects.

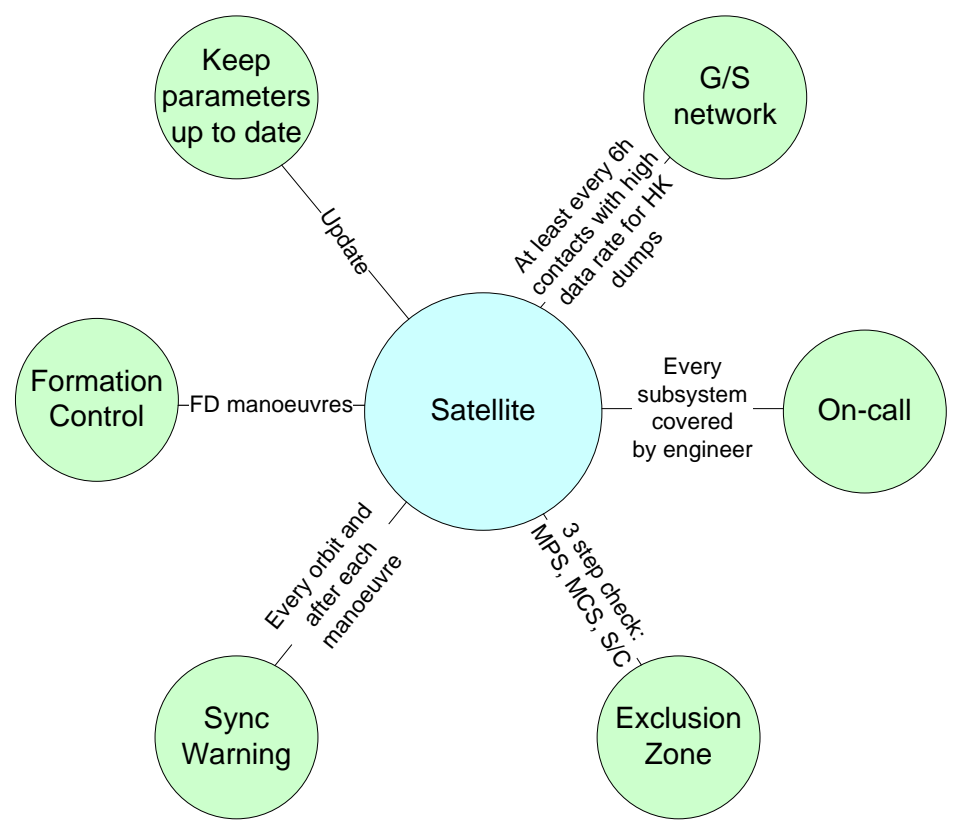


Figure 2. Safe formation flight concept: The main element is the magnet-torquer based safe mode on the satellites that is invoked by the on-board FDIR logic in case of a severe contingency. Complementing the satellite are various ground elements to increase the formation safety and allow a manual reaction as fast as possible. (Abbreviations: MCS: Monitoring and Control System, MPS: Mission Planning System, FD: Flight Dynamics, HK: house-keeping)

\section{A. On-board FDIR concept and ASM-MTQ}

The introduction of the ASM-MTQ mode enables the satellite to stay in thrust-free safe mode long enough for the ground to detect and correct a possible error. Nevertheless there are restrictions: the magnetorquers were originally only designed for the de-saturation of the reaction wheels that act as prime actuators during nominal mission. Due to this fact, the magnetorquers are only strong enough to cope with contingencies that do not exceed certain boundaries; for TSX this means for example that only rotation rates of $<2^{\circ} / \mathrm{s}$ can be handled safely due to the interaction cycle between measurement (magnetometers) and action (torquers). Since this is four times larger than all rates that are commanded on-board, this is sufficient for most problems. Still, there are contingencies that can not be handled; examples are a stuck-open thruster that spins up the satellite or damage of the magnetorquers themselves or their electronics. Therefore, the original thruster based safe mode (ASM-RCS - Reaction Control System) is still in place and serves as last resort in case the satellite gets into life-threatening state. In such a case, e.g. massive power and/or thermal problems due to looking into the sun or deep space, the on-board FDIR logic (Failure, Detection, Isolation and Recovery) triggers the ASM-RCS mode to re-establish a safe attitude - at the risk of illumination/collision.

Since there are various reasons that might lead to such a life-threatening situation, the system answer is actually also more complex than just described. There will be several steps including on-board computer re-boots (to cope for possible problems due to S/W "hang-ups") and the activation of redundant equipments, before the satellite changes back to the ASM-RCS mode, The details are described in Ref. ${ }^{1}$. The availability of the ground stations is an important point concerning the timing aspects of the FDIR concept and is discussed in the next section.

\section{B. Ground station network}

The original plan for the ground station network was based on the assumption that one contact every six hours would be sufficient for operations, especially for the uplink of maneuver commands necessary to keep the orbit and the formation. However, worst case analysis by Astrium showed that after less than two hours the solar panel could be damaged by too low temperatures if constantly looking into deep space. Obviously, a six hour contact scheme would not allow timely ground interaction, even three hours would not be sufficient. Such a dense station network for a high inclination mission would be very expensive, and a tradeoff has to be made between effort and risk. Fortunately the above mentioned case is very unlikely: to begin with, the satellite must somehow be completely stable looking into deep space. Second, when the thrusters are activated there must be enough impulse to reach (and hit!) the other satellite. This also holds true for the danger of flying into the other spacecrafts' illumination (Exclusion Zone). And lastly, the other satellite has to employ its radar just in this moment. All in all, the probability is very low, but still exists in the light of minimal risk considerations and the potential catastrophic result.

After consolidation of the FDIR concept and the definition of the ASM-MTQ mode, it was agreed that the original six hour concept was sufficient to handle the most likely failure cases. Nevertheless improvements were necessary with respect to the data connection and redundancy. The FDIR concept allows the satellite to stay in ASM-MTQ indefinitely but in case of problems as the above mentioned power/thermal problem or too high rotation the thrusters will be activated. Since this case has to be avoided it is of the very important to dump the historic satellite data as quickly as possible to be able to analyze the problem. 
Consequently the ground station network had to be improved in two ways: first, it had to offer enough redundancy in case one contact fails, and second it was necessary to implement redundant, high-rate data lines to transfer the dumped house-keeping data within a few minutes to GSOC. The currently planned ground station network is shown in Figure 3.

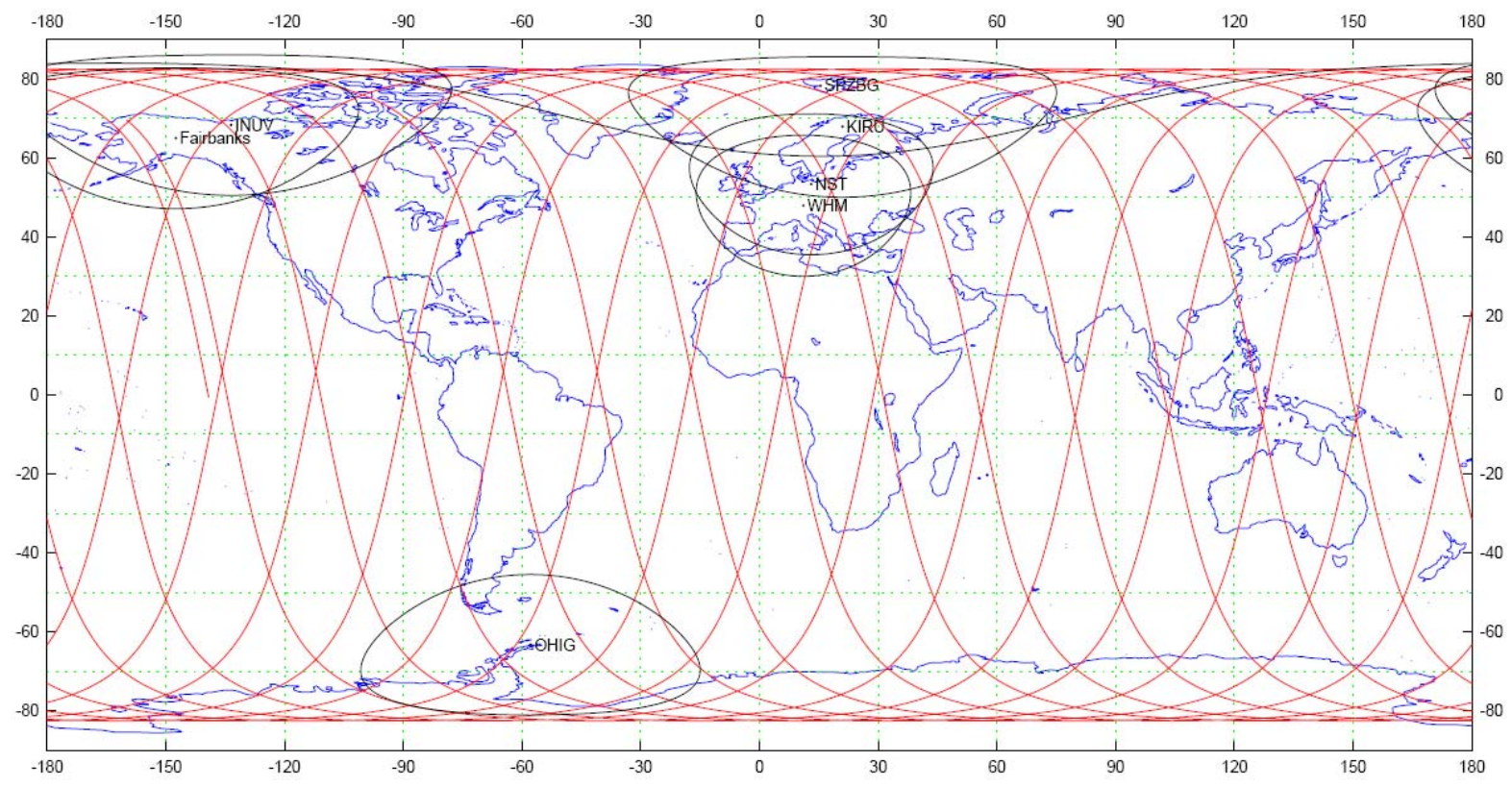

Figure 3. Ground Station Network for the TanDEM-X mission (Abbr: INUV: Inuvik, KIRU: Kiruna, SPZBG: Svalbard, NST: Neustrelitz, WHM: Weilheim, OHIG: O’Higgins)

The primary ground stations for TSX are Weilheim and Neustrelitz in Germany which are DLR owned ground stations. Because of the dusk-dawn orbit these stations cover the early morning and early evening passages. Already in use for additional surveillance are Kiruna (Sweden) and Svalbard (Norway) that allow a 6h contact scheme, although the midnight passes usually have a very low elevation (below $5^{\circ}$ ). For TDX the network is expanded by additional DLR stations in Inuvik (Northern Canada) and O'Higgins (Antarctica). This concept provides sufficient redundancy for all passes:

o Svalbard and/or Kiruna for Weilheim and/or Neustrelitz,

0 Svalbard <-> Kiruna for the noon passes

o O'Higgins <-> Inuvik for the midnight passes (potentially Svalbard as well, but with very low elevation)

Since Inuvik is a new ground station, Fairbanks is an option for the midnight passes in case there are problems but is not integrated right now.

Both satellites are normally operated in parallel with their own dedicated team of operators. However, TSX experience and simulations with dual-satellite operations showed that as soon as there are problems with either or even both satellites it becomes very difficult to maintain parallel operations. Therefore, in case of a contingency it is foreseen that the team takes care of the satellite that has a problem and suspends parallel operation for the time being. It has also been taken care that all ground stations are aware and able to switch support from one to the other satellite on short notice (10 min before the pass) to be able to concentrate on one satellite. 


\section{Formation control}

The orbit control concept of TSX/TDX comprises two steps. First, TSX orbit control maneuvers are planned in order to keep TSX within its $250 \mathrm{~m}$ (radius) tube around its defined Earth-fixed reference orbit. To not affect the TSX/TDX formation, the TSX maneuvers have to be simultaneously replicated by TDX. Secondly, TDX-only maneuvers are planned to reconfigure and maintain the formation with TSX according to the DEM acquisition plan. While the first maneuvers are executed with Hydrazine thrusters, the smaller formation control maneuvers are primarily performed with TDX's cold-gas system. Details on the absolute and relative orbit control concept are given in ${ }^{2}$.

Because of possible maneuver failures (including missed uplink) four problem cases have to be considered when dealing with absolute orbit control maneuvers:

o TSX performs the maneuver, TDX not or partly

o TDX performs the maneuver, TSX not or partly

o the maneuvers are partly executed on both satellites

0 the maneuvers are not executed on both satellites

The last case is without danger, since the (relative) orbit is not affected. However, the other three cases have to be considered within the Flight Dynamics System (FDS). Here, a dedicated software module propagates the TSX and TDX orbit determination results considering all possible maneuver failure scenarios to estimate the minimum distance of the two satellites in the plane perpendicular towards flight-direction. In case a pre-defined threshold (e.g. $50 \mathrm{~m}$ ) could become violated, the planned maneuvers are not released for commanding and the automated maneuver planning process stops. In that seldom case $\left({ }^{3}\right)$ the FD on-call engineer has to cross-check the results and possibly split the maneuver(s) in order to reduce the maneuver size and hence collision risk.

\section{Exclusion zones and sync warnings}

So far it has been described how the close formation is planned to be set up and what problems generally may arise due to unplanned thruster firing. Following that the changes and amendments to the satellite and the ground stations have been discussed and how the formation control is performed. In the next part it shall be described how operations are organized to ensure a maximum degree of safety.

\section{Exclusion Zones}

While FDS checks the maneuver planning to minimise the collision risk, the helix formation as such automatically inherits the danger of illumination. Consequently it has to be ensured that all data-takes are planned in the way that radar activities are prohibited in case one satellite crosses the line of sight of the other. Fortunately, knowing both the SAR instrument beam characteristics and the relative orbit geometry, so-called SAR transmit exclusion zones can be specified for each satellite. For example, from Figure 1 it can be seen that there will be no illumination close to the equator crossings because TSX and TDX are then horizontally separated, but on the way north or south one or the other will block the line of sight.

The exclusion zone concept is shown in Figure 4 and comprises three checks. Initially, the TanDEM-X Acquisition Planner (TAP) specifies the satellite-specific exclusion zone geometry (Exclusion Zone Angles) based on the current helix formation. These contain the forbidden areas/angles in which all radar images are "excluded" to prevent possible damage (see also ${ }^{1}$ ). The TAP also provides an Exclusion Zone Product for satellite upload to assure sufficient reliability (will be explained later). FD receives the exclusion zone angles and calculates from the relative orbit determination results the start and stop times of the Transmit Exclusion Windows (TEW). 
TEWs are provided to the mission planning system (MPS) and to the exclusion zone checker (EZC), where the on-ground checks are performed. During each planning run MPS checks the TEWs and arranges the radar datatakes accordingly. The EZC tool performs the final checking just before the commands are loaded into the monitoring and control system. This allows checking of data-takes planned outside MPS (e.g. maintenance or calibration data-takes) as well as the identification and elimination of wrongly planned data-takes due to an MPS error.

The third and last recipient is the satellite itself to which the exclusion zone information is uploaded directly. It has to be noted however, that this information is not constantly updated and therefore can only be based on ideal formation geometry as considered by the TAP. Orbit control uncertainties are reflected by introducing angular margins, which of course can not account for maneuver failures. The on-board FDIR surveillance thus acts as last resort considering nominal spacecraft operations. It checks the data-takes briefly before and during execution and stops the radar transmission in case of a violation.

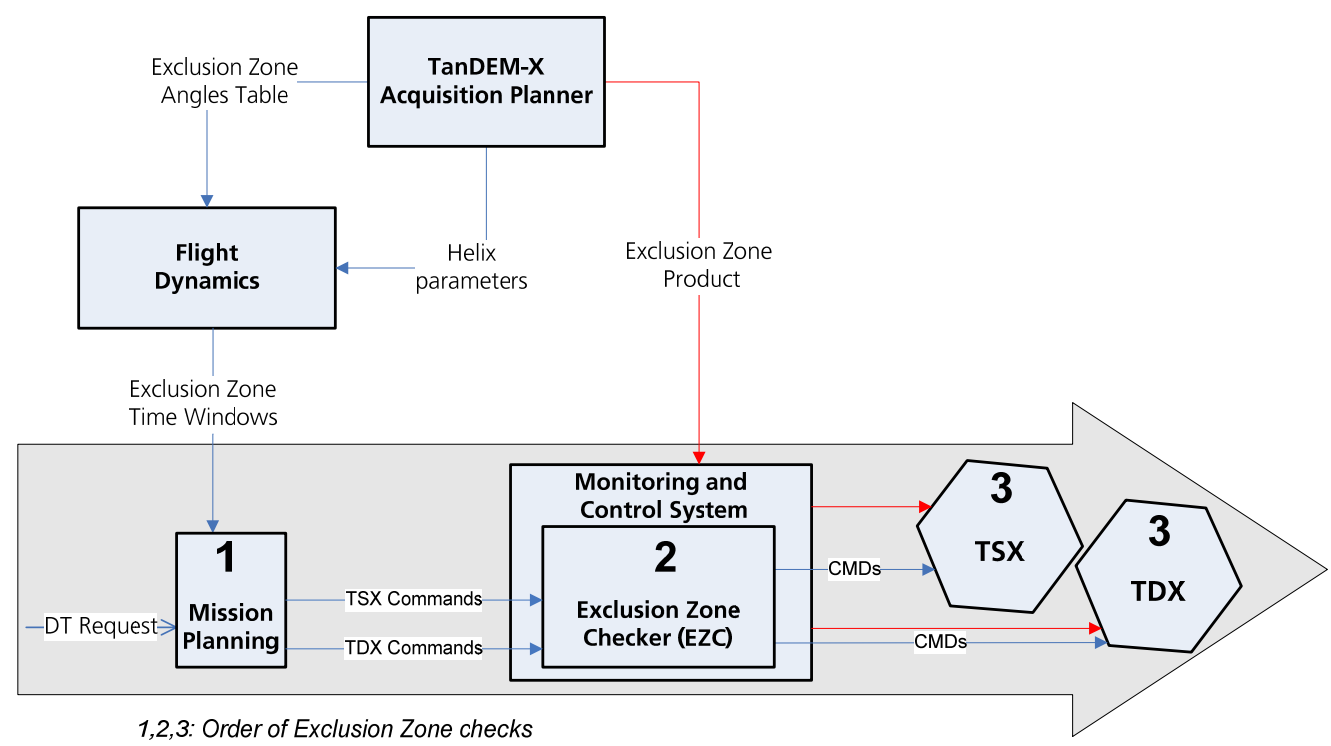

Figure 4. The three stages of the Exclusion Zone Check

Special care has to be taken when maneuvers are planned but not yet executed. Maneuver execution failures as discussed in section $\mathrm{C}$ do not imply a risk of collision but are still large enough to alter the formation and hence the exclusion zones. To handle this, FDS enlarges the Transmit Exclusion Windows after each maneuver to cope with a missed or partly failed maneuver.

During the mission it is foreseen that the helix formation is changed several times to allow for different baselines. Only then, the TAP will provide the respective updates to flight dynamics and the satellites. Since the exclusion zone check only makes sense if the satellites are in close formation, the check is only active within an along-track distance of $10 \mathrm{~km}$.

\section{Sync Warnings}

Exclusion Zones are used to ensure the safety during nominal operations. But what happens if one satellite has a problem and does use the thrusters, even with low impulse. As described previously there might be situations 
where the magnetorquers can not solve the problem (e.g. if the torquers themselves are damaged). Even so it is not likely that the satellites collide (the probability is very low after all) the satellite using its thrusters will definitely leave the pre-defined exclusion zones. To handle this problem an additional safety mechanism is implemented: the sync warnings.

To be able to perform a TanDEM acquisition, it is necessary that TSX and TDX are synchronizing each other. The synchronisation is realized by inserting short radar pulses into the nominal imaging activities using dedicated synchronisation antennas for transmission and reception. Sync warning data-takes are special data-takes using exactly these antennas and that have a low enough power not to damage the other satellite. These data-takes are always sent bi-directional and are evaluated on-board and are independent of exclusion zones. In case one satellite does not receive the expected sync warning pulse of his counterpart, it is interpreted as non-nominal condition of the other satellite. As consequence the position of the other satellite is considered uncertain and to avoid possible illumination the radar transmission is suspended.

\section{E. On-call concept and parameter update}

Complementing the safety concept are two further elements. The TSX On-call concept originally only foresaw one person on-call, the flight operations manager, who then had to decide how to proceed. The experiences made with TSX in the past years lead to the decision to broaden the on-call team in such a way, that not only the flight operations manager (as team lead), but also the main subsystem engineers are on-call. Especially with regard to the quality and complexity of the TanDEM-X mission and its formation aspects this is seen as an important element.

Connected with this is the necessity to always keep on eye on those parameters and configurations that change over time and keep them up to date. Especially when regarding the propulsion systems it is important that parameters that influence the performance are updated. An example for this is the amount of fuel left in the tanks. The emptier the tank is the lower is the tank pressure and the "weaker" is the impulse of the thrusters. If the maneuver calculation is not updated regularly the maneuvers will not have the desired effect. Other examples include the update of on-board FDIR settings. Even so this sounds (and is) trivial in a way, it has to be kept in mind, since once routine operations are established it is easy to delay such work. In a mission like TanDEM-X this should be avoided.

\section{Conclusions}

Originally it was planned to conclude the paper with the first in-orbit experiences of TSX and TDX. Unfortunately, the launch delay prevented the comparison of theory and real life. Still the authors hope that this paper gave an insight on the complexity and challenge of such a project. It should have become clear that even so many improvements have been made a certain risk will always remain. It should also have become clear, that if such a mission is planned the satellite design should be defined accordingly. For the TanDEM-X mission the idea of a close formation mission came only after the TSX design was finished, which meant that there were only few opportunities to adapt the design. Nevertheless we think we found appropriate solutions and we are looking forward to the launch. 


\section{Acknowledgments}

The authors wish to thank J. Herman and B.T. Yugar for their helpful comments and critical reading of the text.

1 Herman, J. et al.; AOCS for TanDEM-X Formation flight at 200m separation in low-Earth orbit; Spaceops 2010, Huntsville

2 Montenbruck O., Kahle R., D'Amico S., Ardaens J.-S.; Navigation and Control of the TanDEM-X Formation; Journal of the Astronautical Sciences, 56(3):341-357 (2008)

3 Kahle R.; TerraSAR-X/TanDEM-X Formation Collision and Illumination Aspects; TD-MOS-TN-4060 (2008)

4 TanDEM-X Ground Segment; Baseline Definition Document; TD-GS-DD-0015 (2007) 